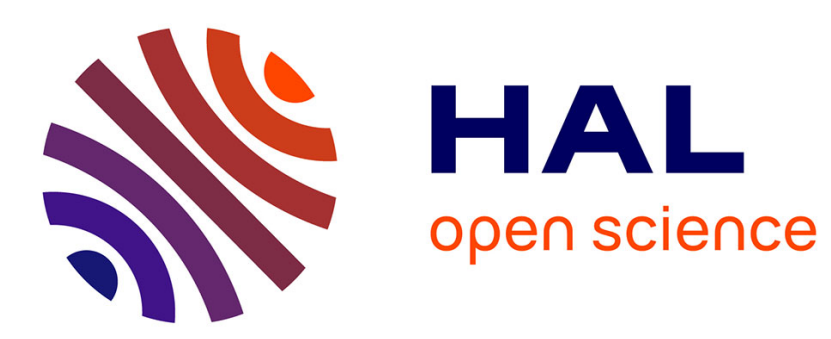

\title{
Conditions of existence of oscillations for hybrid systems
}

Denis Efimov, Wilfrid Perruquetti, Anton Shiriaev

\section{To cite this version:}

Denis Efimov, Wilfrid Perruquetti, Anton Shiriaev. Conditions of existence of oscillations for hybrid systems. IFAC-NOLCOS 2013 - 9th IFAC Symposium on Nonlinear Control Systems, Sep 2013, Toulouse, France. pp.223-228, 10.3182/20130904-3-FR-2041.00092 . hal-00844408

\section{HAL Id: hal-00844408 \\ https://hal.inria.fr/hal-00844408}

Submitted on 15 Jul 2013

HAL is a multi-disciplinary open access archive for the deposit and dissemination of scientific research documents, whether they are published or not. The documents may come from teaching and research institutions in France or abroad, or from public or private research centers.
L'archive ouverte pluridisciplinaire HAL, est destinée au dépôt et à la diffusion de documents scientifiques de niveau recherche, publiés ou non, émanant des établissements d'enseignement et de recherche français ou étrangers, des laboratoires publics ou privés. 


\title{
Conditions of existence of oscillations for hybrid systems
}

\author{
Denis Efimov ${ }^{*, * *, * * * *}$ Wilfrid Perruquetti ${ }^{*, * *}$ \\ Anton Shiriaev ${ }^{* * *, * * * *}$
* Non-A project at INRIA - LNE, Parc Scientifique de la Haute Borne, 40 avenue Halley, Bât.A Park Plaza, 59650 Villeneuve d'Ascq, France, $\{$ Denis.Efimov; Wilfrid.Perruquetti\}@inria.fr ** LAGIS (UMR-CNRS 8146), Ecole Centrale de Lille, BP 48, Cité Scientifique, 59651 Villeneuve-d'Ascq, France
*** Department of Engineering Cybernetics, Norwegian University of Science and Technology, NO-7491 Trondheim, Norway, e-mail: Anton.Shiriaev@itk.ntnu.no
**** Department of Control Systems and Informatics, Saint Petersburg
State University of Information Technologies Mechanics and Optics
(ITMO), Kronverkskiy av. 49, Saint Petersburg, 197101, Russia

\begin{abstract}
The paper extends the notion of oscillations in the sense of Yakubovich to hybrid dynamics. Several sufficient stability and instability conditions for a forward invariant set are presented. The consideration is motivated by analysis of a model of two-link compass-gait biped robot.
\end{abstract}

\section{INTRODUCTION}

Oscillations constitute one of the main operating modes for many systems in nature or in techniques Fransoise [2005], King [2009], Kurths et al. [2007], Burkin et al. [1996], Rogers [1999], Tass [2007]. In some cases it is required to maintain the oscillations, in other cases the oscillations have to be suppressed Chernousko and Fradkov [2000], Fradkov and Pogromsky [1998], Shiriaev and de Wit [2004]. In all cases the conditions of existence of sustained oscillations is of great importance since they allow one to analyze/design a system with desired oscillating properties.

There are many stability theories and definitions of oscillations Fradkov and Pogromsky [1998]. Among them in this work we choose one proposed by Prof. Yakubovich almost 40 years ago Yakubovich [1973]. This approach is rather generic, and it covers periodical and chaotic oscillations. Contrarily to a pure periodical case, when existence conditions are rather sophisticated Mallet-Paret and Sell [1996], the conditions of oscillations in the sense of Yakubovich (Y-oscillations) are simple. For Lurie systems, they are formulated in the frequency domain Yakubovich [1973, 1975], Yakubovich and Tomberg [1989], Burkin et al. [1996]. For a generic nonlinear system, the conditions of Y-oscillations are given using Lyapunov arguments Efimov and Fradkov [2009] or applying homogeneity framework Efimov and Perruquetti [2010]. The main goal of the present paper is to extend the notion of Y-oscillations to hybrid dynam-

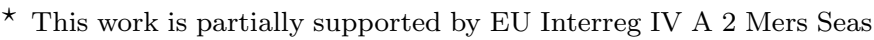
Zeeën Cross-border Cooperation Programme under SYSIASS project 06-020, and by Ministry of Higher Education and Research, NordPas de Calais Regional Council and FEDER through the "Contrat de Projets Etat Region (CPER) CIA 2007-2013".
}

ical systems. For this purpose several stability/instability Lyapunov conditions are formulated for hybrid dynamics.

There exist many applications where the system has a hybrid dynamics (continuous and discontinuous) and it is oscillating. The most important one comes from robot locomotion Freidovich and Shiriaev [2010], Morris and Grizzle [2009], Spong et al. [2006]. The phenomenon has a hybrid nature, due to impacts occurring when a leg is hitting the ground, and the main operating mode is a periodical oscillation. The design/analysis of robot locomotion as a periodically oscillating system is rather sophisticated Freidovich and Shiriaev [2010], Morris and Grizzle [2009], Shiriaev et al. [2010], Shiriaev and de Wit [2004]. However, relaxing the periodicity requirement and considering Y-oscillations, it is possible to develop more constructive conditions for analysis and design of robot motion.

The problem is introduced and illustrated for a twolink compass-gait biped robot model in Section 2. Some preliminaries are given in Section 3. The main result on conditions of Y-oscillations in hybrid systems is presented in Section 4. An example is given in Section 5.

\section{MOTIVATION}

Dynamics of a two-link compass-gait biped robot with a control torque applied at the hip (schematically shown in Fig. 1) can be described by the hybrid system Freidovich and Shiriaev [2010]: 


$$
\begin{gathered}
\dot{x}_{1}=x_{2} ; \\
p_{1} \dot{x}_{2}-p_{2}\left(c_{13} \dot{x}_{4}+s_{13} x_{4}^{2}\right)-p_{4} \sin \left(x_{1}\right)=u ; \\
\dot{x}_{3}=x_{4} ; \\
p_{3} \dot{x}_{4}-p_{2}\left(c_{13} \dot{x}_{2}-s_{13} x_{2}^{2}\right)+p_{5} \sin \left(x_{3}\right)=-u,
\end{gathered}
$$

where $\mathbf{x}=\left[\begin{array}{llll}x_{1} & x_{2} & x_{3} & x_{4}\end{array}\right]^{T} \in \mathbb{R}^{4}$ is the state vector of the robot, $u \in \mathbb{R}$ is the control torque; $s_{13}=\sin \left(x_{1}-x_{3}\right)$, $c_{13}=\cos \left(x_{1}-x_{3}\right)$,

$$
A=\left[\begin{array}{cc}
p_{1}-p_{2} c^{-} & p_{3}-p_{2} c^{-} \\
-p_{2} c^{-} & p_{3}
\end{array}\right], B=\left[\begin{array}{cc}
p_{7} c^{-}-p_{6} & -p_{6} \\
-p_{6} & 0
\end{array}\right],
$$

$c^{-}=\cos \left(x_{1}^{-}-x_{3}^{-}\right) ; C=\mathbb{R}^{4}, D=\left\{\mathbf{x} \in \mathbb{R}^{4}: \cos \left(x_{1}+\right.\right.$ $\left.\psi)=\cos \left(x_{3}+\psi\right)\right\}$ define the sets with continuous and discrete dynamics respectively; $p_{i}, i=\overline{1,7}$ are the robot parameters related with its physical counterparts presented in Fig. 1 as $p_{1}=\left(m_{H}+m\right) l^{2}+m a^{2}, p_{2}=m l b$, $p_{3}=m b^{2}, p_{4}=\left(m_{H} l+m b+m l\right) g, p_{5}=m b g, p_{6}=m a b$, $p_{7}=m_{H} l^{2}+2 m a l ; \psi$ is the slope of the walking surface. The standard abbreviations

$$
x^{-}=x\left(t^{-}\right)=\lim _{\varepsilon \rightarrow 0} x(t-|\varepsilon|), x^{+}=x\left(t^{+}\right)=\lim _{\varepsilon \rightarrow 0} x(t+|\varepsilon|)
$$

are used to denote the values before and after jumps of a solution.

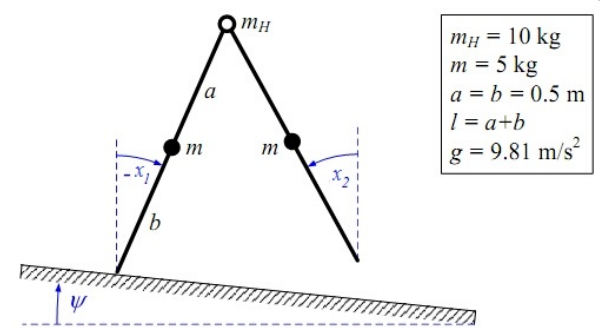

Fig. 1. Schematic of the compass-gait biped on a shallow slope

For $\psi=2.87 \pi / 180$ and $u=0$ the system has two (known) nontrivial periodic solutions Freidovich and Shiriaev [2010]. If the value of $\psi$ is changed or if it is a time-varying signal (that corresponds to a walking on an irregular surface), then the conditions of existence of periodical solutions is an open question. That is more, there is no practically applicable theoretical approach that can address this problem for a time-varying surface slope.

The approaches, for a constant $\psi$, for detection of periodical oscillating modes and their stabilization are largely reported in the literature (see Freidovich and Shiriaev [2010], Morris and Grizzle [2009], Shiriaev et al. [2010], Shiriaev and de Wit [2004] and references therein). This analysis and design methods are very complex and, frequently, only local. The problem complexity is originated by hybrid nature of the dynamics and by complicacy of the periodic motion. It is hard to analyze and stabilize such type of behavior. However, if we would skip the requirement that the motion has to be periodical, allowing other types of oscillating behavior (that is a natural relaxation for a timevarying $\psi$ ), then the oscillation existence conditions could be more simple and global. We would like to demonstrate this developing Y-oscillation concept, which is introduced in the next section.

\section{PRELIMINARY RESULTS}

This section has three parts. The first one deals with the hybrid system formalism introduction following Cai et al. [2007, 2008], Ye et al. [1998]. The second part introduced (pre)asymptotic stability definition and its equivalent Lyapunov characterization from Cai et al. [2008]. The third part is devoted to presentation of Lyapunov sufficient conditions of instability for hybrid systems.

In this work, $\mathbb{R}$ denotes the real numbers, $\mathbb{R}_{+}$the nonnegative real numbers, $\mathbb{Z}$ and $\mathbb{Z}_{+}$are stated for integers and nonnegative integers respectively. The symbol $|\cdot|$ denotes an absolute value for a real scalar or vector, $|\mathbf{x}|_{\mathcal{A}}=\inf _{\mathbf{y} \in \mathcal{A}}|\mathbf{y}-\mathbf{x}|$ is the distance from a point $\mathbf{x} \in \mathbb{R}^{n}$ to a set $\mathcal{A} \subset \mathbb{R}^{n}$. It is said that the function $\alpha: \mathbb{R}_{+} \rightarrow \mathbb{R}_{+}$ belongs to class $\mathcal{K}$ if $\alpha(0)=0$ and it is strictly increasing, it is from class $\mathcal{K}_{\infty}$ if $\alpha \in \mathcal{K}$ and it is radially unbounded.

\subsection{Hybrid systems}

The considered hybrid system has the following form:

$$
\left\{\begin{array}{c}
\dot{\mathbf{x}} \in \mathbf{F}(\mathbf{x}) \quad \mathbf{x} \in C \\
\mathbf{x}^{+} \in \mathbf{G}(\mathbf{x}) \quad \mathbf{x} \in D
\end{array}\right.
$$

where $\mathbf{x} \in \mathcal{O} \subset \mathbb{R}^{n}$ is the state vector (it may contain continuously changing and discrete or logic-based variables); $C \subset \mathcal{O}$ and $D \subset \mathcal{O}$ are the sets where flow or jumps can occur respectively; the (set-valued) maps $\mathbf{F}: \mathcal{O} \rightrightarrows \mathbb{R}^{n}$ and $\mathbf{G}: \mathcal{O} \rightrightarrows \mathbb{R}^{n}$ determine the continuous and discrete dynamics of the system (2). Following Cai et al. [2008] we impose the Standing Assumptions (SAs) on (2):

(SA1) the sets $C \subset \mathcal{O}$ and $D \subset \mathcal{O}$ are relatively closed in $\mathcal{O}$ (i.e. there exist some closed sets $\mathcal{Y}_{C}, \mathcal{Y}_{D}$ such that $D=\mathcal{Y}_{D} \cap \mathcal{O}$ and $\left.C=\mathcal{Y}_{C} \cap \mathcal{O}\right)$;

(SA2) the map $\mathbf{F}: \mathcal{O} \rightrightarrows \mathbb{R}^{n}$ is outer semicontinuous and locally bounded, $\mathbf{F}(\mathbf{x})$ is nonempty and convex for any $\mathbf{x} \in C$;

(SA3) the map $\mathbf{G}: \mathcal{O} \rightrightarrows \mathbb{R}^{n}$ is outer semicontinuous and locally bounded, $\mathbf{G}(\mathbf{x})$ is nonempty subset of $\mathcal{O}$ for any $\mathbf{x} \in D$.

These assumptions allow one to define solutions of the system (2) on hybrid time domains Cai et al. [2007, 2008]. A subset $E \subset \mathbb{R}_{+} \times \mathbb{Z}_{+}$is a compact hybrid time domain if $E=\bigcup_{j=0}^{J-1}\left(\left[t_{j}, t_{j+1}\right], j\right)$ for some finite sequence of times $0 \leq t_{0} \leq \ldots \leq t_{J}$. It is a hybrid time domain if for all $(T, J) \in E, E \cap([0, T] \times\{0,1, \ldots, J\})$ is a compact hybrid time domain. A hybrid arc is a function $\phi: E \rightrightarrows \mathcal{O}$ defined on a hybrid time domain $E$, and such that $\phi(\cdot, j)$ is locally absolutely continuous for each $j$. A hybrid $\operatorname{arc} \phi: \operatorname{dom} \phi \rightrightarrows$ $\mathcal{O}$ is a solution (trajectory) of (2) if $\phi(0,0) \in C \cup D$ and:

(S1) $\dot{\phi}(t, j) \in \mathbf{F}[\phi(t, j)]$ for all $j \in \mathbb{Z}_{+}$and almost all $t \in \mathbb{R}_{+}$such that $(t, j) \in \operatorname{dom} \phi$ and $\phi(t, j) \in C$;

(S2) $\phi(t, j+1) \in \mathbf{G}[\phi(t, j)]$ for all $(t, j+1) \in \operatorname{dom} \phi$ and $\phi(t, j) \in D$.

The conditions $C \cup D=\mathcal{O}$ guarantees existence of solutions for all initial conditions. A solution $\phi$ to the 
hybrid system is called nontrivial if $\operatorname{dom} \phi$ contains at least one point different from $(0,0)$; it is called maximal if its domain cannot be extended. A solution is called complete if its domain is unbounded (complete solutions are maximal), let $\mathcal{S}(\mathbf{x})$ denote the set of all maximal solutions for (2) starting from a point $\mathbf{x} \in \mathcal{O}$. The hybrid system (2) is said to be forward complete on $\mathcal{O}$ if for all $\mathbf{x} \in \mathcal{O}$, each $\phi \in \mathcal{S}(\mathbf{x})$ is complete. The hybrid system (2) is said to be forward invariant on a set $\mathcal{A} \subset \mathcal{O}$ if, for all $\mathbf{x} \in \mathcal{A}$, each $\phi \in \mathcal{S}(\mathbf{x})$ is such that $\phi(t, j) \in \mathcal{A}$ for all $(t, j) \in \operatorname{dom} \phi$ (this definition of forward invariance does not need existence of trajectories for all points of $\mathcal{A}$ ). A point $\mathbf{x}_{0}$ is called an equilibrium of (2) if it is forward invariant for the system.

If $G(D) \cap D \neq \emptyset$, then multiple consequent switches or a Zeno-behavior are possible. In many cases (if $D \subset C$, for example) such a behavior does not correspond to physical nature of the considered system, but it is hard to avoid it in the formulation (2) since the sets $C$ and $D$ are closed. In addition, if $D \subset C$ then on the intersection $C \cap D$ multiple solutions can be initiated, while frequently in practice only a jump is possible in $D$. Let us define the set of maximal solutions initiated from a point $\mathbf{x} \in \mathcal{O}$ without multiple consequent switches with jumps in $D$ as $\hat{\mathcal{S}}(\mathbf{x})$, i.e. $\hat{\mathcal{S}}(\mathbf{x})=\left\{\phi \in \mathcal{S}(\mathbf{x}): t_{j} \neq t_{j+1}, t_{j+1}=\arg _{i n f} \operatorname{it}_{j} \phi(t, j) \in\right.$ $\left.D,\left(t_{j+1}, j+1\right) \in \operatorname{dom} \phi\right\}$. This set can be empty or, if $G(D) \cap D=\emptyset$ then $\hat{\mathcal{S}}(\mathbf{x})=\mathcal{S}(\mathbf{x})$. Further, talking about a solution $\phi$ of the system (2) we will understand a maximal solution $\phi \in \mathcal{S}(\mathbf{x})$ for some $\mathbf{x} \in \mathcal{O}$, except the restriction $\phi \in \hat{\mathcal{S}}(\mathbf{x})$ is explicitly stated.

\subsection{Stability of hybrid systems}

A continuous function $\omega: \mathcal{O} \rightarrow \mathbb{R}_{+}$is proper on $\mathcal{O}$ if $\omega\left(\mathbf{x}_{i}\right) \rightarrow+\infty$ when $\mathbf{x}_{i}$ converges to a boundary of $\mathcal{O}$ or $\left|\mathbf{x}_{i}\right| \rightarrow+\infty$. For a compact set $\mathcal{A} \subset \mathcal{O}$, the function $\omega$ is a proper indicator for $\mathcal{A}$ on $\mathcal{O}$ if it is proper and $\mathcal{A}=\{\mathrm{x} \in \mathcal{O}: \omega(\mathbf{x})=0\}$.

Definition 1. Cai et al. [2008] Let $\mathcal{A} \subset \mathcal{O}$ be compact.

(1) $\mathcal{A}$ is prestable for the system (2) if for each $\varepsilon>0$, there exists $\delta>0$ such that any solution $\phi$ with $|\phi(0,0)|_{\mathcal{A}} \leq \delta$ satisfies $|\phi(t, j)|_{\mathcal{A}} \leq \varepsilon$ for all $(t, j) \in$ $\operatorname{dom} \phi$.

(2) $\mathcal{A}$ is preattractive for the system (2) if there exists $\delta>0$ such that any solution $\phi$ with $|\phi(0,0)|_{\mathcal{A}} \leq \delta$ is bounded with respect to $\mathcal{O}$, and if it is complete, then $\lim _{t+j \rightarrow+\infty}|\phi(t, j)|_{\mathcal{A}}=0$.

(3) $\mathcal{A}$ is preasymptotically stable if it is prestable and preattractive.

(4) $\mathcal{A}$ is asymptotically stable if it is preasymptotically stable and there exists $\delta>0$ such that any maximal solution $\phi$ with $|\phi(0,0)|_{\mathcal{A}} \leq \delta$ is complete.

The set of all $\mathbf{x} \in \mathcal{O}$ from which all solutions are bounded with respect to $\mathcal{O}$ and the complete ones converge to $\mathcal{A}$ is called the basin of preattraction and denoted as $\mathcal{O}_{\mathcal{A}}^{a}$. If the conditions of Definition 1 are valid for any $\delta \in \mathbb{R}_{+}$, then the set $\mathcal{A}$ is globally preattractive/preasymptotically or asymptotically stable for (2).

Definition 2. A compact set $\mathcal{A} \subset \mathcal{O}$ is called NonZeno (NZ) prestable (preattractive/preasymptotically stable/asymptotically stable) for the system (2) if the cor- responding properties of Definition 1 are satisfied for all $\phi \in \hat{\mathcal{S}}$ only.

If for the system (2) the set $\mathcal{A}$ is (pre)asymptotically stable, then it is also NZ (pre)asymptotically stable, the converse is in general false.

Definition 3. Let $\mathcal{X} \subset \mathcal{O}$ be open and $\omega: \mathcal{X} \rightarrow \mathbb{R}_{+}$ be continuous. A function $V: \mathcal{X} \rightarrow \mathbb{R}_{+}$is said to be a smooth Lyapunov function for $(\mathcal{X}, \mathbf{F}, \mathbf{G}, C, D, \omega)$ if there exist $\alpha_{1}, \alpha_{2} \in \mathcal{K}_{\infty}$ such that

$$
\alpha_{1}(\omega(\mathbf{x})) \leq V(\mathbf{x}) \leq \alpha_{2}(\omega(\mathbf{x})) \quad \forall \mathbf{x} \in \mathcal{X}
$$

In this work we will use the following designations:

$$
\begin{aligned}
& \operatorname{Sup} D V(\mathbf{x})=\sup _{\mathbf{f} \in \mathbf{F}(\mathbf{x})}\langle\nabla V(\mathbf{x}), \mathbf{f}\rangle, \\
& \operatorname{Inf} D V(\mathbf{x})=\inf _{\mathbf{f} \in \mathbf{F}(\mathbf{x})}\langle\nabla V(\mathbf{x}), \mathbf{f}\rangle ; \\
& \operatorname{Sup} V^{+}(\mathbf{x})=\sup _{\mathbf{g} \in \mathbf{G}(\mathbf{x}) \cap \mathcal{X}} V(\mathbf{g}), \\
& \operatorname{Inf} V^{+}(\mathbf{x})=\inf _{\mathbf{g} \in \mathbf{G}(\mathbf{x}) \cap \mathcal{X}} V(\mathbf{g}) .
\end{aligned}
$$

This definition does not impose a restriction on derivative or increment of $V$ since both stability and instability properties will be studied below.

Theorem 4. Cai et al. [2008] Let $\mathcal{A}$ be compact and $\mathcal{X}$ be open, $\mathcal{A} \subset \mathcal{X} \subset \mathcal{O}$, and let $\omega$ be a proper indicator for $\mathcal{A}$ on $\mathcal{X}$. The following statements are equivalent for (2):

1) There exists a smooth Lyapunov function $V$ for $(\mathcal{X}, \mathbf{F}, \mathbf{G}, C, D, \omega)$ such that

$$
\begin{aligned}
& \operatorname{Sup} D V(\mathbf{x}) \leq-V(\mathbf{x}) \quad \forall \mathbf{x} \in \mathcal{X} \cap C ; \\
& \operatorname{Sup} V^{+}(\mathbf{x}) \leq e^{-1} V(\mathbf{x}) \quad \forall \mathbf{x} \in \mathcal{X} \cap D
\end{aligned}
$$

and $\mathbf{G}(D \cap \mathcal{X}) \subset \mathcal{X}$.

2) The set $\mathcal{A}$ is preasymptotically stable, its basin of preattraction $\mathcal{O}_{\mathcal{A}}^{a}$ contains $\mathcal{X}$, and $\mathcal{X}$ is forward invariant for $(2)$.

Remark 5. Note that the sufficient part of this theorem holds if the condition (3) is replaced with the following one:

$$
\begin{aligned}
& \operatorname{Sup} D V(\mathbf{x}) \leq-\rho[\omega(\mathbf{x})] \quad \forall \mathbf{x} \in \mathcal{X} \cap C ; \\
& \operatorname{Sup} V^{+}(\mathbf{x}) \leq \lambda V(\mathbf{x}) \quad \forall \mathbf{x} \in \mathcal{X} \cap D,
\end{aligned}
$$

for some function $\rho \in \mathcal{K}$ and a constant $\lambda \in[0,1)$. Indeed, in this case $V[\phi(t, j)] \leq \sigma_{1}\left(V\left[\phi\left(t_{j}, j\right)\right]\right) \sigma_{2}\left(e^{t_{j}-t}\right)$ for all $j \in \mathbb{Z}_{+}$and $t \in\left[t_{j}, t_{j+1}\right]$ such that $(t, j) \in \operatorname{dom} \phi$ and $\phi(t, j) \in C$ for some functions $\sigma_{1}, \sigma_{2} \in \mathcal{K}$; and $V[\phi(t, j+1)] \leq \lambda V[\phi(t, j)]$ for all $(t, j+1) \in \operatorname{dom} \phi$ and $\phi(t, j) \in D$. Therefore, according to Definition 1 the system is prestable and preattractive with respect to the set $\mathcal{A}$ ( $\mathcal{A}$ is preasymptotically stable).

Remark 6. For the sufficient part of Theorem 4 it is enough to have a continuously differentiable or a Lipschitz continuous function $V$ (the same for the results presented below). For a Lipschitz continuous function $V$ the Dini derivatives have to be used. 
Following Ye et al. [1998], a more simple for verification sufficient condition can be proposed for NZ preasymptotical stability of $\mathcal{A}$ based on the following assumption.

Assumption 1. For all $\phi \in \hat{\mathcal{S}}, \sup _{(t, j) \in \operatorname{dom} \phi} t=+\infty$ iff $\sup _{(t, j) \in \operatorname{dom} \phi} j=+\infty$.

These conditions imply that the hybrid time domains of complete solutions in $\hat{\mathcal{S}}$ are unbounded with respect to both $t$ and $j$. This assumption excludes from consideration a case with a finite number of jumps and the Zenobehavior. The reasons for this condition introduction are the following. If the number of switches is finite for a complete solution $\phi \in \hat{\mathcal{S}}$, then the asymptotic transients are predefined by the continuous dynamics only. The Zenobehavior is rather usual in hybrid models, but frequently it has no physical meaning, like in the compass-gait biped robot or the bouncing ball Cai et al. [2008], for instance.

Theorem 7. Let $\mathcal{A}$ be compact and $\mathcal{X}$ be open, $\mathcal{A} \subset \mathcal{X} \subset$ $\mathcal{O}$, and let $\omega$ be a proper indicator for $\mathcal{A}$ on $\mathcal{X}$. Let Assumption 1 be satisfied and there exist a differentiable Lyapunov function $V$ for $(\mathcal{X}, \mathbf{F}, \mathbf{G}, C, D, \omega)$ such that

$$
\operatorname{Sup} D V(\mathbf{x}) \leq 0 \quad \forall \mathbf{x} \in \mathcal{X} \cap C,
$$

$\mathbf{G}(D \cap \mathcal{X}) \subset \mathcal{X}$ and for any solution $\phi \in \hat{\mathcal{S}}$ for all $\left(t_{j+1}, j+\right.$ 1) $\in \operatorname{dom} \phi$ such that $\phi\left(t_{j}, j\right) \in \mathcal{X}$ and $\phi\left(t_{j+1}, j+1\right) \in \mathcal{X}$ for some $\alpha \in \mathcal{K}$

$$
\frac{V\left[\phi\left(t_{j+1}, j+1\right)\right]-V\left[\phi\left(t_{j}, j\right)\right]}{t_{j+1}-t_{j}} \leq-\alpha\left(V\left[\phi\left(t_{j+1}, j+1\right)\right]\right) .(6)
$$

Then the set $\mathcal{A}$ is NZ preasymptotically stable, its basin of preattraction $\mathcal{O}_{\mathcal{A}}^{a}$ contains $\mathcal{X}$, and $\mathcal{X}$ is forward invariant for (2).

All proofs are excluded due to space limitation.

Despite of the conditions of Theorem 7 include the inequality (6) in the time domain for $\phi \in \hat{\mathcal{S}}$, in some cases it is more simple to apply Theorem 7 than Theorem 4 (that we will show later on the examples). Note that without the condition $\operatorname{Sup} V^{+}(\mathbf{x}) \leq V(\mathbf{x}) \quad \forall \mathbf{x} \in \mathcal{X} \cap D$ the function $V$ decreasing may be non-monotone, i.e. $V\left[\phi\left(t_{j+1}, j+\right.\right.$ $1)]>V\left[\phi\left(t_{j+1}, j\right)\right]$ for some $\left(t_{j+1}, j+1\right) \in \operatorname{dom} \phi$ and $\phi\left(t_{j+1}, j\right) \in D$. Therefore, the conditions of Theorem 7 admit a local increasing of $V$ after jumps.

\subsection{Instability of hybrid systems}

This section contains a definition of instability (similarly to Forni and Teel [2010], Ye et al. [1998]) and the corresponding sufficient Lyapunov conditions.

Definition 8. Let $\mathcal{U} \subset \mathcal{O}$ be compact.

(1) $\mathcal{U}$ is preunstable for the system (2) if it is not prestable in the sense of Definition 1, i.e. there exists $\varepsilon>0$ such that for each $\delta>0$ there is a solution $\phi$ with $|\phi(0,0)|_{\mathcal{U}} \leq \delta$ that satisfies $|\phi(t, j)|_{\mathcal{U}}>\varepsilon$ for some $(t, j) \in \operatorname{dom} \phi$.

(2) $\mathcal{U}$ is prerepulsive for the system (2) if there is some $\bar{\delta}>0$ such that for any $0<\delta \leq \bar{\delta}$ there exists a solution $\phi$ with $|\phi(0,0)| \mathcal{U} \leq \delta$ for which there are some $\left(t^{\prime}, j^{\prime}\right) \in \operatorname{dom} \phi$ such that the property $|\phi(t, j)|_{\mathcal{U}}>\bar{\delta}$ holds for all $(t, j) \in \operatorname{dom} \phi$ with $t \geq t^{\prime}, j \geq j^{\prime}$.
(3) $\mathcal{U}$ is strictly prerepulsive for the system (2) if for some $\bar{\delta}>0$ and any solution $\phi$ with $|\phi(0,0)|_{\mathcal{U}} \leq \bar{\delta}$ there exist some $\left(t^{\prime}, j^{\prime}\right) \in \operatorname{dom} \phi$ such that the property $|\phi(t, j)|_{\mathcal{U}}>\bar{\delta}$ holds for all $(t, j) \in \operatorname{dom} \phi$ with $t \geq t^{\prime}$, $j \geq j^{\prime}$.

As in the continuous case Hahn [1967], the hybrid system (2) can be preattractive with respect to a set $\mathcal{A}$ and preunstable with respect to this set simultaneously. If the set $\mathcal{U}$ is prerepulsive for the system (2), then it is preunstable, but this set still can be attractive for a some subset of initial states in a vicinity of $\mathcal{U}$. If it is strictly prerepulsive, then all existent solutions exit a neighborhood of $\mathcal{U}$. The domain of prerepulsion $\mathcal{O}_{\mathcal{U}}^{r}$ contains $\mathbf{x} \in \mathcal{O}$ such that $\phi(0,0)=\mathbf{x}$ and $|\phi(t, j)|_{\mathcal{U}}>\bar{\delta}$ for all $(t, j) \in \operatorname{dom} \phi$ with $t \geq t^{\prime}, j \geq j^{\prime}\left(\left(t^{\prime}, j^{\prime}\right) \in \operatorname{dom} \phi\right)$.

Definition 9. A compact set $\mathcal{U} \subset \mathcal{O}$ is called NZ preunstable (prerepulsive/strictly prerepulsive) for the system (2) if the corresponding properties of Definition 8 are satisfied for $\phi \in \hat{\mathcal{S}}$ only.

If $\mathcal{U}$ is NZ preunstable/prerepulsive for (2), then it is also preunstable/prerepulsive. If $\mathcal{U}$ is strictly prerepulsive, then it is also NZ strictly prerepulsive.

Theorem 10. Let $\mathcal{U} \subset \mathcal{X}$ be compact and $\mathcal{X} \subset \mathcal{O}$ be open, $\mathbf{G}(D \cap \mathcal{X}) \subset \mathcal{X}$. Let a continuous function $\omega: \mathcal{X} \rightarrow \mathbb{R}_{+}$be a proper indicator for $\mathcal{U}$ on $\mathcal{X}$. Let also each $\phi \in \mathcal{S}$ with $\phi(0,0) \in \mathcal{X}$ be nontrivial.

(1) The set $\mathcal{U}$ is strictly prerepulsive for the system (2), its domain of prerepulsion $\mathcal{O}_{\mathcal{U}}^{r}$ contains $\mathcal{X}$, if there exists a differentiable Lyapunov function $U: \mathcal{X} \rightarrow \mathbb{R}_{+}$ for $(\mathcal{X}, \mathbf{F}, \mathbf{G}, C, D, \omega)$ such that for some function $\rho \in \mathcal{K}$ and a constant $\lambda \in[1,+\infty)$

$$
\begin{gathered}
\operatorname{Inf} D U(\mathbf{x}) \geq \rho[\omega(\mathbf{x})] \quad \forall \mathbf{x} \in \mathcal{X} \cap C ; \\
\operatorname{Inf} U^{+}(\mathbf{x}) \geq \lambda U(\mathbf{x}) \quad \forall \mathbf{x} \in \mathcal{X} \cap D .
\end{gathered}
$$

(2) Let Assumption 1 be satisfied. The set $\mathcal{U}$ is $N Z$ strictly prerepulsive for the system (2), its domain of prerepulsion $\mathcal{O}_{\mathcal{U}}^{r}$ contains $\mathcal{X}$, if there exists a differentiable Lyapunov function $U: \mathcal{X} \rightarrow \mathbb{R}_{+}$for $(\mathcal{X}, \mathbf{F}, \mathbf{G}, C, D, \omega)$ such that for any solution $\phi \in \hat{\mathcal{S}}$ for all $\left(t_{j+1}, j+1\right) \in \operatorname{dom} \phi$ such that $\phi\left(t_{j}, j\right) \in \mathcal{X}$ and $\phi\left(t_{j+1}, j+1\right) \in \mathcal{X}$ for some $\alpha \in \mathcal{K}$

$$
\frac{U\left[\phi\left(t_{j+1}, j+1\right)\right]-U\left[\phi\left(t_{j}, j\right)\right]}{t_{j+1}-t_{j}} \geq \alpha\left(U\left[\phi\left(t_{j}, j\right)\right]\right)
$$

and

$$
\operatorname{Inf} D U(\mathbf{x}) \geq 0 \quad \forall \mathbf{x} \in \mathcal{X} \cap C .
$$

Note that the condition (8) is sufficient to prove that the set $\mathcal{U}$ is preunstable for the system (2). Contrarily the condition (7), the conditions (8), (9) do not restrict the Lyapunov function $U$ behavior after switches (it may decrease on $D$ ).

\section{OSCILLATION CONDITIONS}

Extending the results from Efimov and Fradkov [2009], Yakubovich [1973], the definition of Y-oscillations for hybrid systems can be as follows (recall, a function $g: \mathbb{R}^{n} \rightarrow \mathbb{R}$ is called monotone if the condition 
$x_{1} \leq x_{1}^{\prime}, \ldots, x_{n} \leq x_{n}^{\prime}$ implies that everywhere either $g\left(x_{1}, \ldots, x_{n}\right) \leq g\left(x_{1}^{\prime}, \ldots, x_{n}^{\prime}\right)$ or $g\left(x_{1}, \ldots, x_{n}\right) \geq$ $\left.g\left(x_{1}^{\prime}, \ldots, x_{n}^{\prime}\right)\right)$.

Definition 11. Yakubovich [1973], Efimov and Fradkov [2009] A complete solution $\phi \in \mathcal{S}(\mathbf{x})$ with $\mathbf{x} \in \mathcal{O}$ of the system (2) is called $\left[\pi^{-}, \pi^{+}\right]$-oscillation with respect to the output $\psi=\eta(\mathbf{x})$ (where $\eta: \mathcal{O} \rightarrow \mathbb{R}$ is a continuous monotone function) if

$$
\begin{gathered}
\underline{\lim }_{t+j \rightarrow+\infty} \eta[\phi(t, j)]=\pi^{-} ; \overline{\lim }_{t+j \rightarrow+\infty} \eta[\phi(t, j)]=\pi^{+} \\
-\infty<\pi^{-}<\pi^{+}<+\infty .
\end{gathered}
$$

A complete solution $\phi \in \mathcal{S}(\mathbf{x})$ with $\mathbf{x} \in \mathcal{O}$ of the system (2) is called oscillating, if there exist some output $\psi$ and constants $\pi^{-}, \pi^{+}$such that $\phi$ is $\left[\pi^{-}, \pi^{+}\right]$-oscillation with respect to the output $\psi$. A forward complete system (2) is called oscillatory, if for almost all $\mathbf{x} \in \mathcal{O}$ the solutions $\phi \in \mathcal{S}(\mathbf{x})$ of the system are oscillating. The oscillatory system (2) is called uniformly oscillatory, if for almost all $\mathbf{x} \in \mathcal{O}$ for corresponding solutions $\phi \in \mathcal{S}(\mathbf{x})$ there exist the output $\psi$ and the constants $\pi^{-}, \pi^{+}$not depending on initial conditions.

In other words the solution $\phi$ is oscillating if the output $\psi(t, j)=\eta[\phi(t, j)]$ is asymptotically bounded and there is no single limit value of $\psi(t, j)$ for $t+j \rightarrow+\infty$. The term "almost all solutions" is used to emphasize that generally the system (2) has a nonempty set of equilibrium points, thus there exists a set of initial conditions with zero measure such that the corresponding solutions are not oscillating. The notion of oscillations in the sense of Yakubovich is rather generic including periodical oscillations (limit cycles), quasi-periodical, recurrent and chaotic trajectories. The oscillating trajectories could be repelling being oscillating. The trajectories also could be unbounded, it is required to find a function of the state vector $\eta$, that is bounded and admits certain requirements introduced in Definition 11.

Theorem 12. Let $\mathcal{A} \subset \mathcal{X}_{\mathcal{A}}$ and $\mathcal{U} \subset \mathcal{X}_{\mathcal{U}} \subset \mathcal{A}$ be compact and $\mathcal{X}=\mathcal{X}_{\mathcal{A}} \cup \mathcal{X}_{\mathcal{U}} \subset \mathcal{O}$ be open. Let continuous functions $\omega_{\mathcal{A}}: \mathcal{X} \rightarrow \mathbb{R}_{+}, \omega_{\mathcal{U}}: \mathcal{X} \rightarrow \mathbb{R}_{+}$be proper indicators for $\mathcal{A}$ and $\mathcal{U}$ on $\mathcal{X}$ respectively. Let $\mathbf{G}(D \cap \mathcal{X}) \subset \mathcal{X}$, each $\phi \in \mathcal{S}$ with $\phi(0,0) \in \mathcal{X}$ be nontrivial and the system (2) have two Lyapunov functions $V$ and $U$ such that for all $\mathbf{x} \in \mathcal{X}$ :

$$
\begin{aligned}
& v_{1}\left[\omega_{\mathcal{A}}(\mathbf{x})\right] \leq V(\mathbf{x}) \leq v_{2}\left[\omega_{\mathcal{A}}(\mathbf{x})\right] \\
& v_{3}\left[\omega_{\mathcal{U}}(\mathbf{x})\right] \leq U(\mathbf{x}) \leq v_{4}\left[\omega_{\mathcal{U}}(\mathbf{x})\right]
\end{aligned}
$$

$v_{1}, v_{2}, v_{3}, v_{4} \in \mathcal{K}_{\infty}$ and

1) the conditions (4) or (5), (6) with Assumption 1 are valid for the function $V$ on $\mathcal{X}_{\mathcal{A}}$;

2) the conditions (7) or (8), (9) with Assumption 1 are valid for the function $U$ on $\mathcal{X}_{\mathcal{U}}$;

3) $\Omega \cap \Xi=\emptyset$, where $\Omega=\mathcal{A} \cap \mathcal{X}_{\mathcal{U}}$ and $\Xi \subset \mathbb{R}^{n}$ is a set with zero Lebesgue measure containing all equilibriums of the system.

Then the system (2) has an oscillating solution.

Remark 13. If the conditions (4) and (7) are satisfied, then the system (2) is oscillating.

In the next section we will show an example of this theorem application.

\section{EXAMPLE}

Consider the following planar hybrid system:

$$
\mathbf{F}(\mathbf{x})=\left[\begin{array}{c}
(1-|\mathbf{x}|) x_{1}+x_{2} \\
(1-|\mathbf{x}|) x_{2}-x_{1}
\end{array}\right], \mathbf{G}(\mathbf{x})=\left[\begin{array}{c}
x_{2} \\
x_{1}
\end{array}\right]
$$

where $\mathbf{x}=\left[x_{1} x_{2}\right]^{T}, \mathcal{O}=\mathbb{R}^{2}, D=\left\{\mathbf{x} \in \mathcal{O}: x_{1}+k x_{2}=0\right\}$, $C=\mathcal{O}$ and $k \in \mathbb{R} \backslash\{-1,1\}$ is a parameter. Note that in this case $D \subset C$.

The system (10) has the single equilibrium at the origin for $k \neq \pm 1(\Xi=\{0\})$. Next, it is easy to verify that the Lyapunov function $W=|\mathbf{x}|^{2}$ for the continuous dynamics F has the derivative $\dot{W}=2(1-\sqrt{W}) W$ for all $\mathbf{x} \in \mathbb{R}^{2}$, then $W\left(t, t_{0}, W_{0}\right)=\left[1-\left(1-W_{0}^{-0.5}\right) e^{t_{0}-t}\right]^{-2}$ for any initial condition $W\left(t_{0}\right)=W_{0}$, which according to Efimov and Fradkov [2009], Efimov and Perruquetti [2010] implies existence of oscillating trajectories into the set $W(\mathbf{x})=1$ (for $\dot{\mathbf{x}}=\mathbf{F}(\mathbf{x})$ ) and forward completeness of (10). To prove that for any initial condition $\mathbf{x}\left(t^{\prime}, j\right) \in \mathcal{O}, t^{\prime} \in \mathbb{R}_{+}, j \in \mathbb{Z}_{+}$ for solutions of the system (10) always there exists a time instant $t^{\prime \prime} \geq t^{\prime}$ such that $\mathbf{x}\left(t^{\prime \prime}, j\right) \in D$ assume that it is not, then from the expression of $W\left(t, t_{0}, W_{0}\right)$ given above the trajectories of the continuous dynamics converge to the set where $W(\mathbf{x})=1$ and the continuous dynamics is reduced to a linear oscillator, thus $t^{\prime \prime}$ exists and Assumption 1 is satisfied.

For the chosen $\mathbf{G}$ we have that $W\left(\mathbf{x}^{+}\right)=W(\mathbf{x})$ for all $\mathbf{x} \in D$, therefore the value of $W$ is not changing for the discrete dynamics. In addition, for $k \neq \pm 1$ the set $D \backslash\{0\}$ is not invariant for (10), therefore for the almost all instants of time the behavior of $W$ is predefined by the continuous dynamics.

To apply Theorem 12 we can choose the origin as the set $\mathcal{U}, U(\mathbf{x})=W(\mathbf{x})$ and $\mathcal{X}_{\mathcal{U}}=\{\mathbf{x} \in \mathcal{O}:|\mathbf{x}|<1\}$, then $U(t, j)$ is a strictly increasing function on $\mathcal{X}_{\mathcal{U}}$ and for any solution $\phi \in \hat{\mathcal{S}}$ of $(10)$ with $\phi(0,0) \in \mathcal{X}_{\mathcal{U}}$ we have $U\left[\phi\left(t_{j+1}, j+1\right)\right]-$ $U\left[\phi\left(t_{j}, j\right)\right] \geq\left(t_{j+1}-t_{j}\right) 2\left(1-\sqrt{U\left[\phi\left(t_{j}, j\right)\right]}\right) U\left[\phi\left(t_{j}, j\right)\right]$ for all $\left(t_{j}, j\right) \in \operatorname{dom} \phi$ while $\phi$ rests in $\mathcal{X}_{\mathcal{U}}$, i.e. the conditions of Theorem 10 are satisfied.

Let us select the set $\mathcal{A}=\{\mathbf{x} \in \mathcal{O}:|\mathbf{x}| \leq 1\}$, then $\mathcal{X}_{\mathcal{A}}=\mathcal{O} \backslash \mathcal{A}$. Using the function $W$ we can prove that this $\mathcal{A}$ is NZ preasymptotically stable for (10). Indeed, for any solution $\phi \in \hat{\mathcal{S}}$ of $(10)$ with $\phi(0,0) \in \mathcal{X}_{\mathcal{A}}$ we have $W\left[\phi\left(t_{j+1}, j+1\right)\right]-W\left[\phi\left(t_{j}, j\right)\right] \leq\left(t_{j+1}-t_{j}\right) 2(1-$ $\left.\sqrt{W\left[\phi\left(t_{j+1}, j+1\right)\right]}\right) W\left[\phi\left(t_{j+1}, j+1\right)\right]$ for all $\left(t_{j}, j\right) \in \operatorname{dom} \phi$ while $\phi$ rests in $\mathcal{X}_{\mathcal{A}}$. Select $V(\mathbf{x})=\max \{0, W(\mathbf{x})-1\}$, then outside of the set $\mathcal{A}$ we have $V\left[\phi\left(t_{j+1}, j+1\right)\right]-$ $V\left[\phi\left(t_{j}, j\right)\right] \leq-\left(t_{j+1}-t_{j}\right) \alpha\left\{V\left[\phi\left(t_{j+1}, j+1\right)\right]\right\}$ for $\alpha(s)=$ $-(1-\sqrt{1+s})(1+s) \in \mathcal{K}$ and all conditions of Theorem 7 are verified.

The set $\Omega=\{\mathbf{x} \in \mathcal{O}:|\mathbf{x}|=1\}$ does not contain the equilibrium of (10). Therefore, all conditions of Theorem 12 are satisfied and the system (10) has an oscillating solution.

The system trajectories for different values of $k$ are shown in Fig. 2. As we can deduce from these results of simulation, the system stay oscillating for all values of $k$, but the types of the oscillating trajectories differ signif- 
icantly (there exist two disjoint oscillating subsets in $\Omega$ for $k=|1.5|$ and a discontinuous limit cycle for $k=|0.5|$ ).
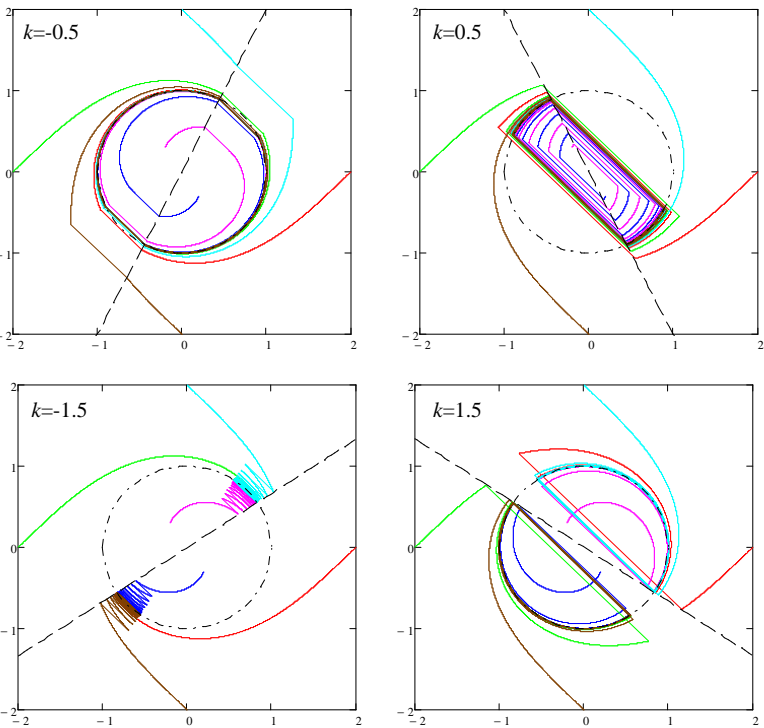

Fig. 2. The hybrid system $\{-0.5,0.5,-1.5,1.5\}$

\section{CONCLUSIONS}

The paper develops the framework of oscillations in the sense of Yakubovich to the class of hybrid systems (having continuous and discrete dynamics). Some sufficient Lyapunov conditions for stability/instability of hybrid systems are presented. The utility of the proposed theory is demonstrated on an example. Extensions to different robotic systems are planned in future works.

\section{REFERENCES}

I.M. Burkin, G.A. Leonov, and A.I. Shepelyavyi. Frequency Methods in Oscillation Theory. Mathematics and Its Applications. Kluwer, Dordrecht, 1996. in Russian: 1992.

C. Cai, A.R. Teel, and R. Goebel. Smooth lyapunov functions for hybrid systems part i: Existence is equivalent to robustness. IEEE Trans. Autom. Control, 52(7):12641277, 2007.

C. Cai, A.R. Teel, and R. Goebel. Smooth lyapunov functions for hybrid systems part ii: (pre)asymptotically stable compact sets. IEEE Trans. Aut. Contr., 53(3): 734-748, 2008.

F.L. Chernousko and A.L. Fradkov, editors. Control of Oscillations and Chaos: 2000 2nd International Conference Proceedings. IEEE, November 2000.

D. Efimov and A.L. Fradkov. Oscillatority of nonlinear systems with static feedback. SIAM J. Control Optimization, 48(2):618-640, 2009.

D. Efimov and W. Perruquetti. Oscillations conditions in homogenous systems. In Proc. IFAC NOLCOS Symp. pages 1379-1384, Bologna, 2010.

Fulvio Forni and Andrew R. Teel. Instability and overshoots of solutions for a class of homogeneous hybrid systems by lyapunov-like analysis. In Proc. 49th IEEE
Conference on Decision and Control (CDC), pages 2390-2395, Atlanta, 2010.

A.L. Fradkov and A.Yu. Pogromsky. Introduction to oscillations and chaos. World Scientific, Singapore, 1998.

Jean-Pierre Fransoise. Oscillations En Biologie: Analyse Qualitative Et Modeles. Springer-Verlag, August 2005.

L. Freidovich and A.S. Shiriaev. Transverse linearization for an underactuated compass-like biped robot and analysis of the closed-loop system. In Proc. the 8th IFAC Symposium on Nonlinear Control Systems (NOLCOS), Bologna, August 2010. IFAC.

W. Hahn. Stability of Motion. Stability of Motion, NY, 1967.

George C. King. Vibrations and Waves. John Wiley \& Sons Inc., August 2009.

Jurgen Kurths, Grigory V. Osipov, and Changsong Zhou. Synchronization in Oscillatory Networks. Springer Series in Synergetics. Springer-Verlag, September 2007.

J. Mallet-Paret and G.R. Sell. The poincar-bendixson theorem for monotone cyclic feedback systems with delay. J. Differential Equations, 125:441-489, 1996.

B. Morris and J.W. Grizzle. Hybrid invariant manifolds in systems with impulse effects with application to periodic locomotion in bipedal robots. IEEE Transaction on Automatic Control, 54(8):1751-1764, 2009.

Graham Rogers. Power System Oscillations. The Kluwer International Series in Engineering and Computer Science. Kluwer Academic Pub, 1999.

A.S. Shiriaev and C. Canudas de Wit. Virtual constraints: a tool for orbital stabilization of nonlinear systems theory. In Proc. 6th IFAC Symposium NOLCOS 2004, pages 1355-1360, Stuttgart, 2004. IFAC.

A.S. Shiriaev, L. Freidovich, and S. Gusev. Computing a transverse linearization for mechanical systems with two and more underactuated degrees of freedom. IEEE Transactions on Automatic Control, 55(4):893906, 2010.

M. W. Spong, S. Hutchinson, and M. Vidyasagar. Robot Modeling and Control. John Wiley \& Sons, New York, 2006.

Peter A. Tass. Phase Resetting in Medicine and Biology: Stochastic Modelling and Data Analysis. Springer Series in Synergetics. Springer-Verlag, January 2007.

V.A. Yakubovich. Frequency oscillations conditions in nonlinear systems with stationary single nonlinearity. Siberian Math. J., 14(2), 1973.

V.A. Yakubovich. Oscillations in systems with discontinuous and hysteresis nonlinearities. Automation and Remote Control, 12, 1975.

V.A. Yakubovich and E.A. Tomberg. Conditions for self-induced oscillations in nonlinear systems. Siberian Math. J., 30:641-653, 1989.

H. Ye, A.N. Michel, and L. Hou. Stability theory for hybrid dynamical systems. IEEE Trans. Aut. Contr., 43(4): 461-475, 1998. 